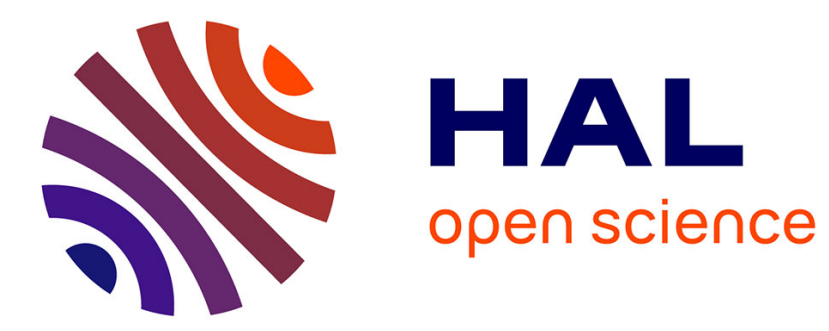

\title{
Data Processing using Artificial Neural Networks to Improve the Simulation of Lung Motion
}

Rémy Laurent, Michel Salomon, Julien Henriet, Marc Sauget, Régine Gschwind, Libor Makovicka

\section{- To cite this version:}

Rémy Laurent, Michel Salomon, Julien Henriet, Marc Sauget, Régine Gschwind, et al.. Data Processing using Artificial Neural Networks to Improve the Simulation of Lung Motion. Biomedical Engineering: Applications, Basis and Communications, 2012, 24 (6), pp.563 - 571. 10.4015/S1016237212500524 . hal-00940605

\section{HAL Id: hal-00940605 https://hal.science/hal-00940605}

Submitted on 2 Feb 2014

HAL is a multi-disciplinary open access archive for the deposit and dissemination of scientific research documents, whether they are published or not. The documents may come from teaching and research institutions in France or abroad, or from public or private research centers.
L'archive ouverte pluridisciplinaire HAL, est destinée au dépôt et à la diffusion de documents scientifiques de niveau recherche, publiés ou non, émanant des établissements d'enseignement et de recherche français ou étrangers, des laboratoires publics ou privés. 


\title{
Data Processing using Artificial Neural Networks to Improve the Simulation of Lung Motion
}

\author{
R. Laurent ${ }^{\dagger, *}$, M. Salomon ${ }^{\ddagger}$, J. Henriet $^{\dagger}$, M. Sauget, R. Gschwind ${ }^{\dagger}$, and L. Makovicka ${ }^{\dagger}$ \\ ${ }^{\dagger}$ Chrono-Environnement Laboratory, UMR 6249 CNRS \\ University of Franche-Comté \\ BP 71427, Pôle Universitaire des Portes du Jura \\ 25211 Montbéliard Cedex, France, \\ ${ }^{\ddagger}$ FEMTO-ST Institute, UMR 6174 CNRS \\ University of Franche-Comté \\ BP 527, Rue Engel Gros \\ 90016 Belfort Cedex, France
}

Received Day Month Year

Revised Day Month Year

\begin{abstract}
To optimize the delivery in lung radiation therapy, a better understanding of the tumor motion is required. On the one hand to have a better tumor-targeting efficiency, and on the other hand to avoid as much as possible normal tissues. The 4D-CT allows to quantify tumor motion, but due to artifacts it introduces biases and errors in tumor localization. Despite of this disadvantage, we propose a method to simulate lung motion based on data provided by the $4 \mathrm{D}-\mathrm{CT}$ for several patients. To reduce uncertainties introduced by the 4D-CT scan, we conveniently treated data using artificial neural networks. More precisely, our approach consists in a data augmentation technique. The data resulting from this processing step are then used to build a training set for another artificial neural network that learns the lung motion. To improve the learning accuracy, we have studied the number of phases required to precisely describe the displacement of each point. Thus, from 1118 points scattered across 5 patients and defined over 8 or 10 phases, we obtained 5800 points of 50 phases. After training, the network is used to compute the positions of 40 points from five other patients on 10 phases. These points allow to quantify the prediction performance. In comparison with the original data, the ones issued from our treatment process provide a significant increase of the prediction accuracy: an average improvement of $16 \%$ can be observed. The motion computed for several points by the neural network that has learnt the lung one exhibits an hysteresis near the one given by the $4 \mathrm{D}-\mathrm{CT}$, with an error smaller than $1 \mathrm{~mm}$ in the cranio-caudal axis.
\end{abstract}

Keywords: Respiratory motion prediction, Computer simulation, 4D-CT, Data processing.

\section{INTRODUCTION}

The aim of radiation therapy is to provide the maximum radiation dose to a tumor, while delivering the lowest dose possible to the surrounding normal tissues. In case of lung tumors it is crucial to be able to track them precisely, otherwise the respiratory motion will be a major problem for the treatment process. A modality that allows to follow the position of lung tumors is $4 \mathrm{D}$ CT imaging, nevertheless motion artifacts and other biases can degrade the acquired data. Today, 4D-CT has been adopted for routine clinical use; therefore, we propose a method based on these data. Note that we do not want to find the locations given by the $4 \mathrm{D}-\mathrm{CT}$, since the motion artifacts would be still present, but we rather want a smooth realistic and regular motion simulation.

The fourth dimension in a scanner enables temporal synchronization of respiratory acquisition. The quality of the resulting images depends mainly on two parameters: the period of the breathing for a patient, which can greatly vary due to the stressful situation; the rotation time of the tube that can range from 0.5 to 1 second,

\footnotetext{
* Corresponding author: Dr. Laurent, Chrono-Environnement, UMR 6249 CNRS, University of Franche-Comté, BP 71427, Pôle Universitaire des Portes du Jura, 25211 Montbéliard Cedex, France. Tel.: +33-3811994682; Fax: +33-381994610; E-mail: remy.laurent@pu-pm.univ-fcomte.fr.
} 
leading to kinetic blur. Usually, for each table position, the respiratory cycle is represented in 10 uniformly distributed "phases", each phase corresponding to an instant in the cycle. These images are then combined to produce the final scan, more precisely the different images corresponding to a same phase are concatenated together to form a single image. Thus, several respiratory cycles are needed and the slices corresponding to a 3D image at a specific phase might not belong to it. The phase values range from $0 \%$ to $100 \%$, where $0 \%$ and $50 \%$ represent respectively the maximum exhalation and inspiration. Furthermore, since the respiratory cycle is periodic, $0 \%$ and $100 \%$ define the same phase.

Figure 1 shows the respiratory signal measured by RPM system (Varian) during the acquisition of a 4DCT scan. The moments of data acquisition (beam on), the variations in the breathing period, and the phases calculated by RPM software are determined using the RPM respiration data file. The successive upper dots on the respiratory signal identify the $0 \%$ phases, while the lower ones correspond to the $50 \%$ phases. The figure also describes how the image for the $50 \%$ phase is built by concatenation of images obtained on several respiratory periods.

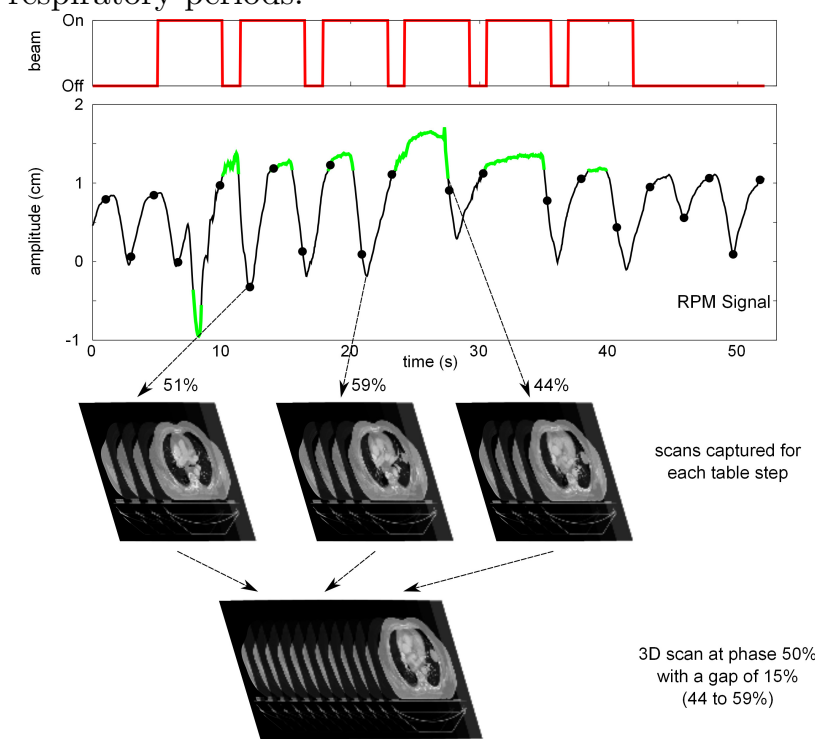

Fig. 1 4D-CT imaging: reconstruction process of a 3D CT image at a discrete phase (50\% in this example) for a patient according to its respiratory signal

The analysis of Fig. 1 shows that the patient's ability to achieve reproducible respiratory signals has a great impact on the temporal distribution of the phases. Thus, although phases 51, 59, and $44 \%$ are close to the target phase $50 \%$, the corresponding amplitudes recorded by the RPM system are very different (a gap of more than $1.25 \mathrm{~cm}$, whereas the average respiratory amplitude is $1.5 \mathrm{~cm}$ ). These differences explain the kinetic artifacts observed on the superior/inferior axis, they are easily detected by the discontinuity in the anatomical structures being imaged.

As an illustration, the analysis of $504 \mathrm{D}-\mathrm{CT}$ from different patients, presented in Ref. 19, has highlighted the presence of artifacts around the diaphragm or heart in $90 \%$ of the images. The impact on the measure of the tumor has been determined by Sarker et al. ${ }^{13}$. Indeed, they established correlated variations of lung volume and discontinuities in the acquired $4 \mathrm{D}$ respiratory signal. Furthermore, using several maximal inspirations, they have measured for the average position of the center of the tumor mass a standard-deviation of $1 \mathrm{~cm}$ for a difference of $59.7 \%$ in lung volume. In fact, the smaller the tumor dimensions, the larger the error on the position of its center.

Kinetic blurs appear in the axial plane, they are characterized by a poor definition of anatomical structures. This kind of artifacts is not negligible, since Persson et al. ${ }^{12}$ observed that, in addition to kinetic artifacts, blurring affects GTV (Gross Tumor Volume) delineation. Indeed, the authors have shown that the variation in GTV size throughout respiratory phases results from partial volume effects caused by kinetic blur due to large tumor motion. Together, kinetic artifacts and blurs lead to contouring error of the GTV, with size differences up to $90 \%$.

Consequently, although $4 \mathrm{D}-\mathrm{CT}$ is a commonly used modality, it leads to artifacted images because it fails to adapt to respiratory variations (period and magnitude), and also requires some time to record the data. Moreover, the spatial resolution is another limiting factor for the selection of anatomical landmarks. Hence, in spite of the fact that $4 \mathrm{D}-\mathrm{CT}$ provides a measure, we can and we want to improve the quality and the accuracy of the motion information for each patient. This paper presents an original approach for real-time simulation of lung motion using a neural network. It focuses on the data processing method based on additional neural networks to improve the quality of the $4 \mathrm{D}-\mathrm{CT}$ data used to learn the motion.

To simulate the lung motion, various approaches have already been proposed. Sarut et al. ${ }^{14}$ and Villard ${ }^{17}$ designed an algorithm based on continuum mechanics formalism. They studied the lung deformations induced by the diaphragm and the rib-cage, and obtained a subvoxel accuracy. However, as they build a three dimensional image using a model defined by many complex equations, their method has a high computational cost. Furthermore, some exams are required in order to carefully measure all tissue elasticity parameters. This approach can give an accurate prediction of 
the lung motion, but is not well suited for routine clinical use. Boldea et al. ${ }^{2,3}$ simulated a $4 \mathrm{D}$ scan during a respiratory cycle using two scanner images: one at the very beginning and another one at the very end of the inspiration. Nevertheless both precision and computation time are very similar to the former methods. The approach proposed by Hostettler et al. ${ }^{8}$, which is based both on reference marks on patient skin and one scan, is interesting since it permit a low-dose exposition. Unfortunately, it provides a low accuracy: 5 to $7 \mathrm{~mm}$ whatever the voxel dimensions are.

Studies also focused on the determination of the hysteresis of lung motion. In fact, a respiratory model that exhibits an hysteretic behavior of lung would allow to improve the accuracy in radiation therapy. Lung tumors are also prone to hysteresis ${ }^{15,18}$, hence this phenomenon must be considered during the treatments. In Ref. 11, a respiratory motion simulator taking into account this useful information has been built. It offers 5 degrees of freedom: tissues location at a specific phase, the tidal volume and airflow according to the breathing phase, and like the methods described previously uses 4D-CT scans. The gap between the measured and predicted points ranges from 0.75 to $1.55 \mathrm{~mm}$.

Data augmentation techniques have already been used in many domains. Indeed, they represent an invaluable tool for Bayesian statistics, particularly to investigate posterior distribution using Markov chain Monte Carlo methods ${ }^{7}$. An expectation-maximization algorithm can also benefit from data augmentation ${ }^{5,20}$. The relevance of increasing a limited data set in the context of Artificial Neural Networks (ANN) has been also studied in Ref. 6. In this work, we consider such an approach to effectively increase the size of the dataset and reduce the influence of artifacts and bias introduced by 4D-CT imaging. Precisely, the initial small and noisy dataset is used to train neural networks, from which a larger set is then built. In this way, we obtain smoothed data that allow to greatly improve the learning of lung motion by another neural network.

\section{MATERIALS AND METHODS}

\section{Dataset construction}

To simulate a patient's lung motion, we have proposed to use an artificial neural network that learns the motion of some anatomic characteristic points during the respiratory cycle ${ }^{10}$. The dataset used to train the neural network through a holdout validation approach is generated by plotting the motion of points in 10 discrete and regular phases of the breathing cycle in $4 \mathrm{D}-$ CT images from 5 patients (patients 0 to 4 ). The five remaining patients (patients 5 to 9) are used as a testing dataset. The characteristic points were determined by a radiation therapist in non-pathogenic areas and thus only the motion of normal tissues will be considered. This process leads to several biases and noise in the data: on the one hand, the phase in which a point is plotted is not always displayed by the scanner, and on the other hand the resolution of the CT images limits the accuracy of the plotted points. In fact, the considered 4D-CT images were obtained using different scanning devices and protocols, the points are marked in a system whose resolution is $1 \times 1 \times 2 \mathrm{~mm}^{3}$ and $1 \times 1 \times 2.5 \mathrm{~mm}^{3}$. Table 1 shows the distribution of the 1518 points present in our dataset among the 10 patients. Two facts about the dataset should be noted. Firstly, patients 4, 7, 8, and 9 data subsets come from POPI-model developed by Vandemeulebroucke et al. ${ }^{16}$. Secondly, two phases are missing for patient 0: phases $10 \%$ and $80 \%$.

Table 1 Dataset points distribution

\begin{tabular}{|c|c|c|c|c|c|}
\hline Patient\# & 0 & 1 & 2 & 3 & 4 \\
\hline Lung vol. & 4.62 & 2.86 & 5.48 & 2.60 & 5.94 \\
\hline Pts/Phase & 21 & 25 & 24 & 8 & 38 \\
\hline Phases & {$[0 ; 20-70 ; 90]$} & {$[0-90]$} & {$[0-90]$} & {$[0-90]$} & {$[0-90]$} \\
\hline Total & 168 & 250 & 240 & 80 & 380 \\
\hline Patient\# & 5 & 6 & 7 & 8 & 9 \\
\hline Lung vol. & 3.10 & 4.42 & 3.72 & 4.91 & 4.05 \\
\hline Pts/Phase & 6 & 4 & 10 & 10 & 10 \\
\hline Phases & {$[0-90]$} & {$[0-90]$} & {$[0-90]$} & {$[0-90]$} & {$[0-90]$} \\
\hline Total & 60 & 40 & 100 & 100 & 100 \\
\hline
\end{tabular}

\section{Multilayer perceptron neural networks}

To reduce all potential sources of error and increase the number of points in the dataset, the plotted points are fitted using neural networks. The chosen network model is the feedforward MultiLayer Perceptron (MLP), which is a type of artificial neural network well-known for its universal approximation property ${ }^{4}$. We selected the classical single hidden layer topology. This one is composed of sigmoid-type hidden neurons, while the output neurons have a linear activation function. Our network has 4 inputs: the three dimensional coordinates of a point at phase $0 \%$ and the phase number for which its location must be predicted, and the output vector is also a three dimensional vector. For each point, the best network architecture is determined through an incremental approach ${ }^{1}$ (successive addition of a a neuron into the hidden layer), according to the motion complexity. Thus, we obtain for each point a regular and 
smooth description of its trajectory, which does not depend on the scanner resolution and is less noisy. Moreover, we can complement the dataset with missing information (for example, we can add the two missing phases for patient 0 ) or with a finer description of motion, since the displacement of each point can be obtained for any phase. Thanks to this data processing, the learning set is less noisy and more realistic, which allows to improve the performance of the ANN learning the lung motion. Figure 2 describes the different steps of our approach: an appropriate data treatment through a collection of neural networks is done, followed by the motion learning using another ANN, these steps represent the learning process, then the resulting (trained) network is only used to predict the lung motion (normal execution).

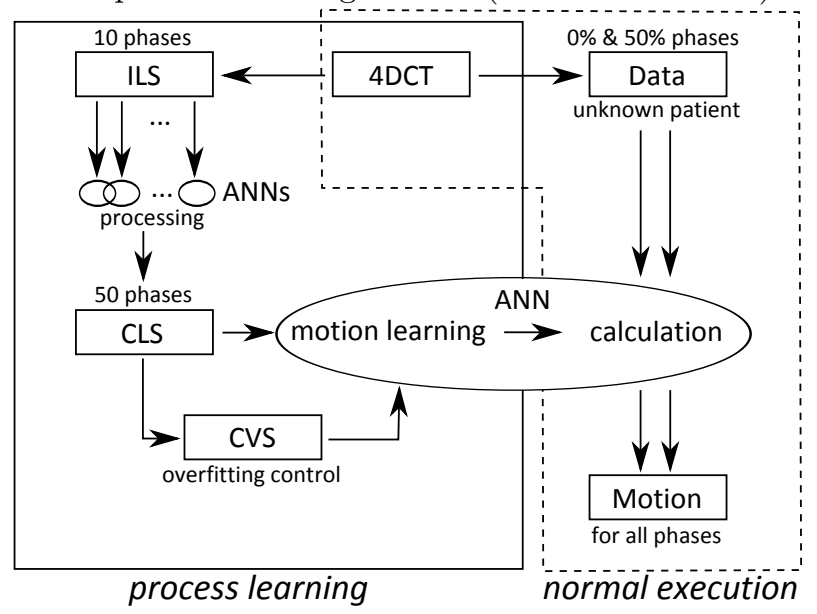

Fig. 2 Schematic description of the proposed approach $($ ILS $=$ Initial Learning Set; CLS $=$ Corrected Learning Set; CVS $=$ Corrected Validation Set)

The neural network developed to learn the displacements of the lung is also a single hidden layer MLP. It is composed of neurons with activation functions (hidden / output) identical to those of the network model described above. The network has 8 inputs : the three dimensional coordinates of a point at both phases $0 \%$ and $50 \%$, the lung volume to identify the patient, and finally the phase number for which the neural network must predict the point location. The network is trained through supervised learning by feeding it with the data resulting from the processing. More precisely, the training updates the synaptic weights and the biases of the neurons in order to minimize the Mean Squared Error (MSE) between the network outputs and the expected ones defined by the data. Hence, the training process consists in applying an optimization algorithm in order to minimize the MSE. In this work, we have chosen the self-scaling Limited-memory BFGS algorithm, a quasiNewton algorithm ${ }^{9}$, in combination with Wolfe's linear search. It uses the classical backpropagation algorithm to calculate the gradient of the error. An incremental approach allows to fix the number of neurons in the hidden layer ${ }^{1}$. Five network trainings were performed using data from 5 patients, these trainings differ from each other by the data used as validation set for overfitting control (holdout validation): each time the data from a different patient are considered. As long as the mean squared error obtained for the validation set decreases the training improves the network, whereas a long term increase means a loss of the generalization ability.

The resulting networks usually have 8 neurons on the hidden layer. Therefore, we decided to retain this topology for the remainder of our study. Our method to control overfitting consists in performing the training 1000 iterations (also called epochs) beyond the one for which a minimum MSE is achieved for the validation set. More precisely, 1000 iterations allow to verify that a divergence of several millimeters is observed for the validation set after the last minimum. After the study of the optimal number of phases to learn the lung motion, a comparison of different trainings will be done. This comparison will focus on the impact of both learning and validation sets quality: ILS and CLS will, respectively, denote the Initial and Corrected Learning Set, while IVS and CVS will represent their counterparts for the Validation Set. Let us note that the validation set will also be used as test set to assess the performances.

\section{RESULTS}

Once a training is completed, the network computes the displacements of the points belonging to one patient over 100 phases. In the less favorable case, which corresponds to patient 4 , the average computation time is $205 \mathrm{~ms}( \pm 32)$ on a typical personal computer (dualcore CPU running at $2.1 \mathrm{GHz}$ ). That means an almost real-time simulation of lung motion, since less than $3 \mathrm{~ms}$ are required to compute the positions of the 38 points.

\section{Data processing}

To ensure a good continuity between the adjacent simulated phases $99 \%$ and $0 \%$, the phase domain is extended between $-20 \%$ and $120 \%$. Since the studied phenomenon is periodic, a correspondence between the phases of this new domain must be done. However, due to bias in some data, particularly between phases $90 \%$ and $0 \%$, continuity is not fully guaranteed. For example, Fig. 3(b) shows that, according to the $4 \mathrm{D}-\mathrm{CT}$ images, the point has a trajectory that covers $5 \mathrm{~mm}$ between two consecutive phases at the end of the exhalation. In other words, 
a large movement is observed when the displacement of a point is theoretically very small. This gap is reduced to less than $1.8 \mathrm{~mm}$, thanks to the processing.

Figure 3 shows the noisy trajectories resulting from $4 \mathrm{D}-\mathrm{CT}$ for points belonging to patients 0 (points 1 and 2; plots (a) and (b)) and 4 (point 3; plot (c)), and the smoothed ones obtained by our processing. The trajectories are given considering the $z$ cranio-caudal axis. As a reminder, the points of patient 0 are only defined on 8 phases: phases $10 \%$ and $80 \%$ are missing. In particular, it can be seen that the displacement of point 1 is very noisy at phase $50 \%$, clearly showing that data smoothing is relevant. In all cases, the smoothing induced by our processing does neither lead to a loss of information on the movement, nor alter the information. So, we obtain a more accurate and smoother description of the displacement of a point, which in consequences improves the convergence of the artificial neural network learning the lung motion.

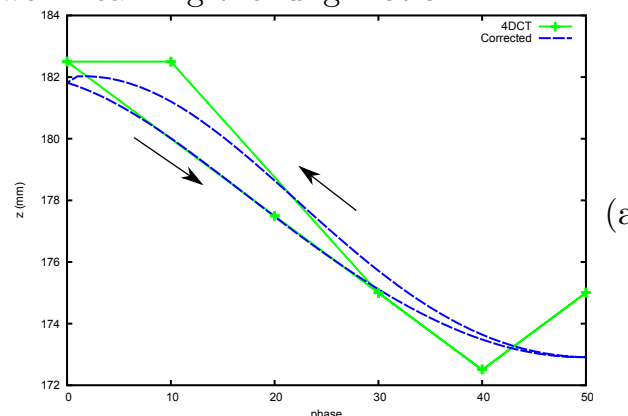

(a)

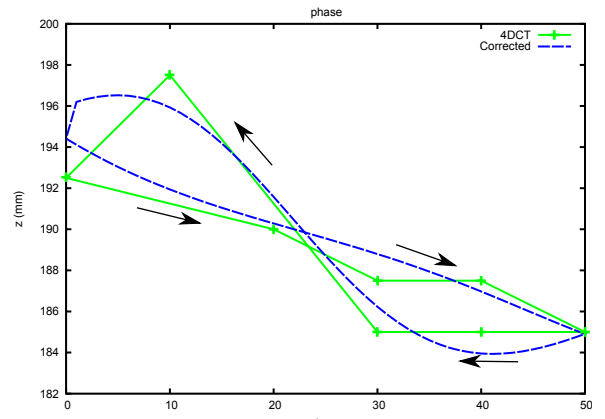

(b)

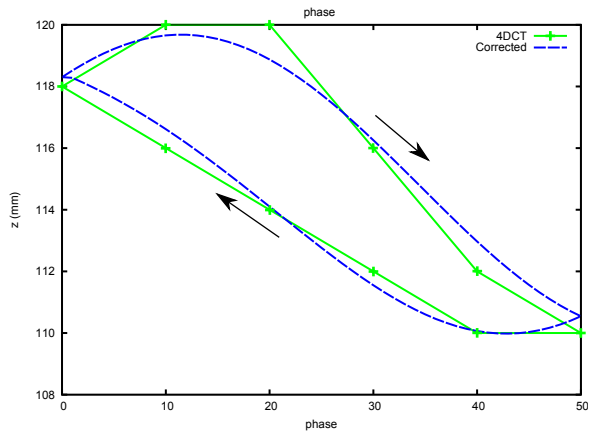

Fig. 3 Processing impact on the motion of the three different points

Figure 3 also describes the periodic displacement of the different points. Indeed, since phase $100 \%$ corre- sponds to the $0 \%$ one, a point should come back to its initial position. Note that in this figure phases 10, 20,30 , and $40 \%$ give the point position for them and also, respectively, for phases $90,80,70$, and $60 \%$. Hence, this figure shows in addition to the bias between phases $90 \%$ and $0 \%$, the hysteresis-like motion, for both $4 \mathrm{D}$ $\mathrm{CT}$ and smoothed data. Consequently, the ANN learning the lung motion uses the data without any a priori information.

\section{Optimizing the training process}

In the following results, the ones corresponding to patient 1 are not given, because of the patient characteristics: the smallest lung volume and its points are the most scattered inside the lungs. The lung motion of patient 1 can be simulated, but we would obtain an approximative simulation because there is no similar case known.

The results are given according to the patient considered as the validation set to control overfitting, since we use a cross-validation process. Also, we do not give any indication on the trainings convergence because the training sets are quite different. We show the results gained once the trainings are performed. Thus, they present for the patient used as validation set the average distance between the expected positions and the ones predicted by the ANN for all phases. To compare the treated data with the originals, only 10 phases are considered to compute the average error, except for patient 0 which is defined by 8 phases. This last point explains why the errors for this patient are the smaller: a missing phase $(80 \%)$ exhibits an important gradient of motion. Table 2 clearly highlights the impact of the missing phases.

Table 2 is devoted to the study of the number of phases used to describe the points motion according to the validation patient. Several observations can be made. First, patient 2 is characterized by a stable accuracy whatever the number of learnt phases, which is a consequence of the point locations: they are localized in a lung area with a simple motion. Secondly, conversely patient 4 has the largest errors because it is the patient that has the biggest lung volume. In this case the ANN extrapolates, since it predicts movements beyond the ranges of the training dataset. However, the neural network is able to correctly simulate the motion (with larger errors) of each point. Thirdly, the ideal number of phases is 50, whereas 100 phases induce a loss of generalization ability reflected by the increase of the average errors. In the following, we will retain the 50 phases setup for the motion description. 


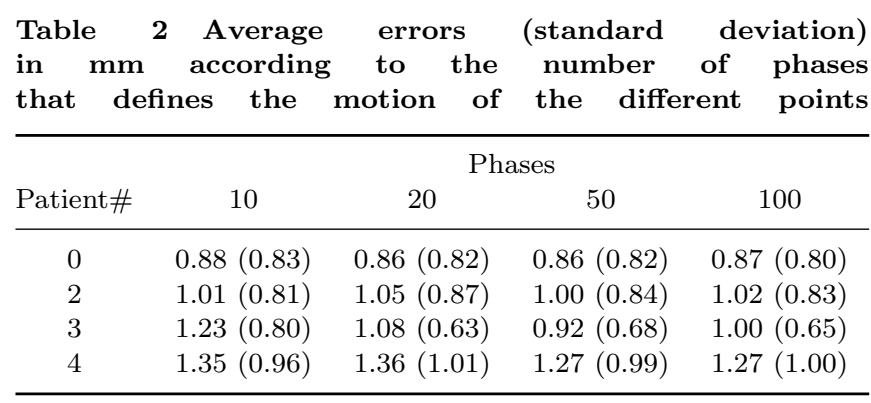

Similarly, table 3 shows the evolution of the average error according to the training and validation sets for each test patient. Considering ILS trainings and both types of validation/test sets the noise resulting from each patient becomes quantifiable. Note that even without any data processing an artificial neural network smoothes the noise. This first comparison shows that the patient data are noisy, with a noise level that is not negligible: the average distance ranges from $3 \%$ to $8 \%$. The neural network is not able to fully reduce the impact of the noise because we have a small learning set due to few patients. Therefore, a data processing is required. The last column, for each patient, in table 3 assesses the relevance of the processing on the learning set: both bias and noise are reduced such that the neural network converges to a smaller learning error. The estimated accuracy improvement between ILS/IVS and CLS/CVS varies between $16 \%$ and $42 \%$ according to the patient.

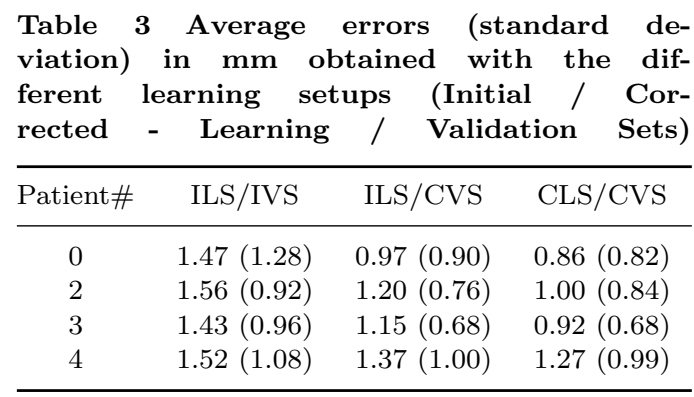

\section{Simulation of the lung motion}

As we can see in table 3, CLS/CVS is the optimal training setup for our neural network, so it was to retained to learn the lung motion. The neural network used is the one obtained with patient 2 as validation set. In this case the 1518 points of the dataset are used as follows:

- the learning subset is composed of 4600 points (patients $0,1,3$, and $4 ; 50$ phases);
- patient 2 (1200 points) defines the validation subset;

- the remaining 400 points (patients 5 to 9; 10 phases) correspond to the test samples.

Thus, the learning, validation, and testing subsets represent, respectively, about $74 \%, 20 \%$, and $6 \%$ of the whole dataset. Let us emphasize that the learning and validation points have been processed to improve the network training, whereas it is not the case of the test samples. Indeed, in normal execution the neural network is supposed to deal with original $4 \mathrm{D}-\mathrm{CT}$ data. Table 4 presents the results obtained for the test patients for the initial dataset (ILS/IVS) and the ones (CLS/CVS) issued from our treatment method. The mean improvement of the prediction accuracy is about $16 \%$ and reaches $30 \%$ for patient 9 .

Table 4 Average errors (standard deviation) in $\mathrm{mm}$ obtained for the different test patients (Initial / Corrected - Learning / Validation Sets)

\begin{tabular}{ccl}
\hline Patient\# & ILS/IVS & CLS/CVS \\
\hline 5 & $1.15(0.68)$ & $1.02(0.62)$ \\
6 & $1.45(0.55)$ & $1.25(0.72)$ \\
7 & $2.30(2.32)$ & $2.00(2.26)$ \\
8 & $1.37(0.96)$ & $1.16(0.83)$ \\
9 & $2.18(1.49)$ & $1.53(1.15)$ \\
\hline
\end{tabular}

To validate our approach, we carried out a comparison of the results with a linear approximation of the motion. This approximation is obtained considering that the displacements remain constant whatever the phase, by applying a linear regression between the maximum exhalation and expiration phases $(0 \%$ and $50 \%)$, and thus without taking into account the hysteresis.

Obviously, the linear interpolation leads to a symmetrical motion between the phase ranges $0-50 \%$ and $50-100 \%$. Figure 4 shows the motion predicted for two distinct points issued from test subset, the first one belongs to patient 6 and the second to patient 8 , the trajectories obtained by linear regression and from $4 \mathrm{D}-\mathrm{CT}$ data are also plotted. We can notice that linear regression is not accurate enough to describe the motion. Indeed, as highlighted by the 4D-CT the motion gradient depends on the phase. By comparison with the linear approximation, our approach that considers hysteresis is more competitive, closer to the $4 \mathrm{D}-\mathrm{CT}$. 


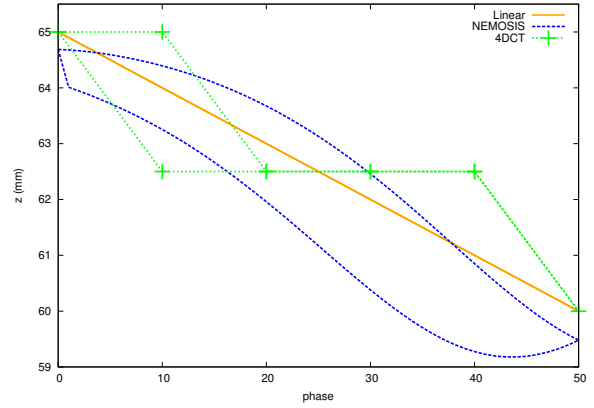

(a)

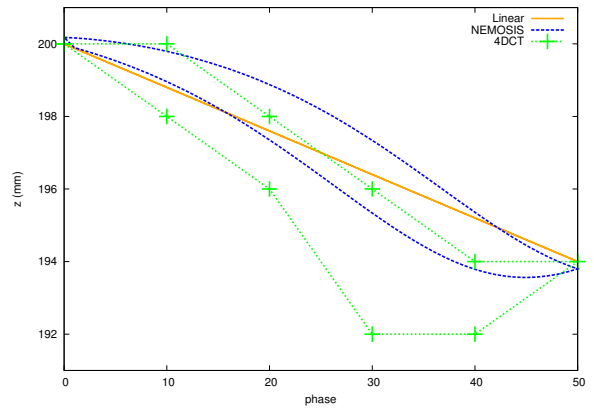

(b)

Fig. 4 Motion simulated for two points, each belonging to a different patient

This observation can also be made from Fig. 5, which presents the average prediction errors for the points belonging to patient 8 . The data from phases $0 \%$ and $50 \%$ were not taken into account to compute the values presented in the histogram, because their errors for the linear regression are zero by construction. The positions predicted by our neural network are, on average, the nearest to the ones given by the $4 \mathrm{D}-\mathrm{CT}$. The lower standard deviations reflect the better fitting of the neural network, thanks to the simulation of the hysteresis, as suggested by Fig. 4. The error becomes favorable for the linear regression when the point motion is constant on $0-50 \%$ or $50-100 \%$, or even for the whole phases. In that case, the neural network still simulates a small movement and thus gives an error. For the set of points from patient 8 , the average error of the positions predicted by the ANN is, in comparison with the $4 \mathrm{D}-\mathrm{CT}$ images, less than $2 \mathrm{~mm}$ (except point 2), which means lower than the $\mathrm{z}$ axis image resolution.

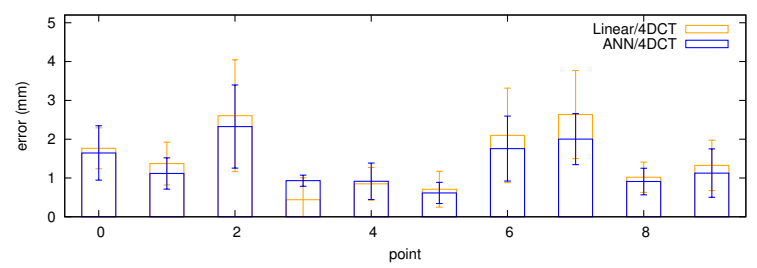

Fig. 5 Neural network versus linear regression: average prediction errors for all points of patient 8 , considering all phases

\section{DISCUSSION}

Table 1 shows great variations in the errors according to the patient. In fact, our method is sensitive to the lung volume, since the largest errors for patients 7 , and 9 result from lung volumes distant from the ones of the patients used as learning and validation data. Obviously, a neural network can only give suitable predictions for unknown inputs when they are similar to some samples used during the training step. As a consequence, patients 5, 6 and 8, which have lung volumes of the same order than several training patients (see table 1 ), have the lowest errors.

When a $4 \mathrm{D}-\mathrm{CT}$ produces a point trajectory without hysteresis (see Fig. 4(a)), the artificial neural network simulates ones thanks to its ability to generalize from the given patients. More generally, for both points presented in Fig. 4 the trajectories predicted by the ANN reflect the $4 \mathrm{D}-\mathrm{CT}$ ones, taking into account motion artifacts and hysteresis. The minimum position of these points is located between phases $50 \%$ and $60 \%$, while we considered that phase $50 \%$ was the maximum inspiration. Note that we had no a priori knowledge on the points positions, but rather the data used to train the network have their minimum at phase $50 \%$ or $60 \%$.

Our method is based on $4 \mathrm{D}-\mathrm{CT}$ data and we have seen previously that these data contain artifacts. Consequently, finding suitable displacements for the different points is more difficult. Despite this, our approach uses this tool to accurately simulate the motion of points whatever the phase and considering hysteresis.

\section{CONCLUSION AND FUTURE WORK}

The simulation of lung motion is a difficult challenge, because breathing is a complex phenomenon which requires to consider, in addition to hysteresis, the various types of deformation induced by the different areas of the lung. Our approach consists in learning the lung motion using some patients data, so that the breathing of the unknown patients can be accurately simulated. Thus, once the data is pretreated to significantly reduce the noise resulting from the 4D-CT artifacts, we can produce in a realistic way for normal tissues, every deformation that belongs to our domain of definition (currently it does not cover the whole lung, due to lack of data). We have shown that the 4D-CT scans are noisy and that the proposed processing allows to improve the convergence of the neural network designed to learn the lung motion. An average accuracy improvement of $16 \%$ was observed. The results of our method are not limited by any constraint posed by the 4D-CT modality (such 
as a voxel size of $1 \times 1 \times 2.5 \mathrm{~mm}^{3}$ ) or the only knowledge of ten discrete phases with an important uncertainty.

In the future, we plan a clinical evaluation so that we can differentiate the patients' sex, pathologies, and tissues in order to improve our approach for a possible use in clinical routine practice. Indeed, our method runs in almost real-time and thanks to a simultaneous acquisition of a patient respiratory phase, we will compute each time the tumors contours. To finalize our method, we will also extend the domain of definition to cover the whole lung by considering more points and more patients.

\section{ACKNOWLEDGEMENTS}

The authors thank the LCC (Ligue Contre le Cancer), Région Franche-Comté and the PMA (Pays de Montbéliard Agglomération) for their financial support.

\section{REFERENCES}

1. Jacques M. Bahi, Sylvain Contassot-Vivier, and Marc Sauget. An incremental learning algorithm for function approximation. Advances in Engineering Software, 40(8):725-730, 2009.

2. V. Boldea, D. Sarrut, G. Sharp, and S. Jiang. 4D-CT lung motion models construction with deformable registration: estimation of motion nonlinearity and hysteresis. Med Phys, 35(3):1008-1018, 2008.

3. Vlad Boldea. Intégration de la respiration en radiothérapie: apport du recalage déformable d'images. Phd thesis, Université Lumière Lyon 2, 2006.

4. George Cybenko. Approximation by superpositions of a sigmoidal function. Mathematics of Control, Signals and Systems, 2:303-314, 1989.

5. Chris Fraley. On computing the largest fraction of missing information for the EM algorithm and the worst linear function for data augmentation. Computational Statistics \&3 Data Analysis, 31(1):13 - 26, 1999.

6. Iffat A. Gheyas and Leslie S. Smith. A neural networkbased framework for the reconstruction of incomplete data sets. Neurocomputing, 73(16-18):3039 - 3065, 2010. 10th Brazilian Symposium on Neural Networks (SBRN2008).

7. Gavin J. Gibson, George Streftaris, and Stan Zachary. Generalised data augmentation and posterior inferences. Journal of Statistical Planning and Inference, 141(1):156 - 171, 2011.

8. Alexandre Hostettler, Stéphane A. Nicolau, Luc Soler, C. Forest, and Yves Rémond. Real time simulation of organ motions induced by breathing: first evalution on patient data. In Biomedical simulation: 3rd International symposium, pages 9-18, 2006.

9. William W. Hsieh. Machine Learning Methods in the Environmental Sciences - Neural Networks and Kernels. Cambridge university press, 2009.
10. R. Laurent, J. Henriet, M. Salomon, M. Sauget, F. Nguyen, R. Gschwind, and L. Makovicka. Utilisation d'un réseau de neurones artificiels pour la simulation des mouvements pulmonaires. Cancer/Radiothérapie, 15(2):123 - 129, 2011.

11. Daniel A. Low, Parag J. Parikh, Wei Lu, James F. Dempsey, Sasha H. Wahab, James P. Hubenschmidt, Michelle M. Nystrom, Maureen Handoko, and Jeffrey D. Bradley. Novel breathing motion model for radiotherapy. International Journal of Radiation Oncology*Biology*Physics, 63(3):921 - 929, 2005.

12. Gitte Fredberg Persson, Ditte Eklund Nygaard, Carsten Brink, Jonas Westberg Jahn, Per Munck af Rosenschöld, Lena Specht, and Stine Sofia Korreman. Deviations in delineated GTV caused by artefacts in 4DCT. Radiotherapy and Oncology, 96(1):61 - 66, 2010.

13. Joyatee Sarker, Alan Chu, Kit Mui, John A. Wolfgang, Ariel E. Hirsch, George T. Y. Chen, and Gregory C. Sharp. Variations in tumor size and position due to irregular breathing in 4d-ct: A simulation study. Medical Physics, 37(3):1254-1260, 2010.

14. David Sarrut, S. Delhay, Pierre-Frédéric Villard, Vlad Boldea, Michael Beuve, and Patrick Clarysse. A comparison framework for breathing motion estimation methods from 4-D imaging. IEEE Trans. Med. Imaging, 26(12):1636-1648, 2007.

15. Yvette Seppenwoolde, Hiroki Shirato, Kei Kitamura, Shinichi Shimizu, Marcel van Herk, Joos V. Lebesque, and Kazuo Miyasaka. Precise and real-time measurement of $3 \mathrm{D}$ tumor motion in lung due to breathing and heartbeat, measured during radiotherapy. International Journal of Radiation Oncology*Biology*Physics, 53(4):822 - 834, 2002.

16. J. Vandemeulebroucke, D. Sarrut, and P. Clarysse. Point-validated pixel-based breathing thorax model. In International Conference on the Use of Computers in Radiation Therapy (ICCR), Toronto, Canada, jun 2007.

17. Pierre-Frédéric Villard. Simulation du Mouvement Pulmonaire pour un Traitement Oncologique. Phd thesis, Université Claude Bernard, 2006.

18. H. Wu, M. Langer, D. Demir, E.M. Shmukler, Q. Zhao, N. Gammon, and H. Shirato. Hysteresis analysis of lung tumor motion in radiation treatment. International Journal of Radiation Oncology*Biology*Physics, 72(1, Supplement 1):S443 - S444, 2008. Proceedings of the American Society for Therapeutic Radiology and Oncology 50th Annual Meeting, American Society for Therapeutic Radiology and Oncology 50th Annual Meeting.

19. Tokihiro Yamamoto, Ulrich Langner, Billy W. Loo Jr., John Shen, and Paul J. Keall. Retrospective analysis of artifacts in four-dimensional CT images of 50 abdominal and thoracic radiotherapy patients. International Journal of Radiation Oncology*Biology*Physics, 72(4):1250 - 1258, 2008.

20. Yang Yang, Ira M. Longini Jr., and M. Elizabeth Halloran. A data-augmentation method for infectious disease incidence data from close contact groups. Computational Statistics $\&$ Data Analysis, 51(12):6582 - 6595, 2007. 